Furthermore, Kliem et al reported a larger confidence interval for the overall effect size. The confidence interval that they calculated corresponds well to the respective interval that we reported in Table 1, which was $0.26-0.83 .{ }^{1}$ A narrower confidence interval, however, was erroneously reported by us in the forest plot owing to a transcription error (Fig. 2, 95\% CI $0.41-0.67)^{1}-$ see correction.

Second, after excluding the study by Bateman \& Fonagy, ${ }^{3}$ which they regarded as an outlier, Kliem et al reported a fail-safe $N$ of 16 unpublished studies with an effect size of 0 , which would need to be added in the meta-analysis to change the result from significant to non-significant. However, we could not replicate these findings. After excluding the study by Bateman \& Fonagy, we found a fail-safe $N$ of 69 , which is above $55(5 K+10)$, again indicating that the effect is robust. Apparently, Kliem et al erroneously did not calculate a fail-safe $N$ according to Rosenthal but according to Orwin's method. ${ }^{4}$ Consequently, they did not assess how many studies with ES $=0$ had to be included to change the result from significant to non-significant but to 'not significantly different from $0.16^{\prime}$ - an irrelevant result.

Third, results of Bayesian meta-analyses depend largely on the specification of prior assumptions on the treatment effect and between-trial variance. Since Kliem et al did not provide any information about the assumptions of their analyses, it is impossible to interpret the presented result reasonably.

In summary, we could not confirm the discrepancies reported by Kliem et al. We did not find substantial heterogeneity or any cogent indication of publication bias. The effect in favour of long-term psychodynamic psychotherapy was confirmed as robust. Instead, we could show that most of those 'discrepancies' seem to be based on differing methodological approaches.

1 Leichsenring F, Rabung S. Long-term psychodynamic psychotherapy in complex mental disorders: update of a meta-analysis. Br J Psychiatry 2011; 199: $15-22$.

2 Hedges LV, Vevea JL. Fixed- and random-effects models in meta-analysis. Psychol Meth 1998; 3: 486-504.

3 Bateman A, Fonagy P. The effectiveness of partial hospitalization in the treatment of borderline personality disorder, a randomized controlled trial. Am J Psychiatry 1999; 156: 1563-9.

4 Orwin RG. A fail-safe $\mathrm{N}$ for effect size in meta-analysis. J Educ Behav Stat 1983; 8: 157-9.

Falk Leichsenring, Department of Psychosomatics and Psychotherapy University of Giessen, Ludwigstrasse 76, 35392 Giessen, Germany. Email: Falk.Leichsenring@psycho.med.uni-giessen.de

doi: $10.1192 /$ bjp.200.5.429a

\section{Identifying responders in randomised controlled trials for depression}

The usefulness of antidepressants in patients with mild or moderate depression has been questioned and therefore Thase et al $^{1}$ re-analysed randomised controlled trial (RCT) data for escitalopram. They calculated that a subgroup of patients (14\%) with mild or moderate depression did respond to treatment and not to placebo, but that the subgroup of patients with severe depression who responded to treatment was larger (23\%).

The findings from Thase et al ${ }^{1}$ illustrate what is known from the philosophy of science about RCTs: if $\mathrm{T}$ causes $\mathrm{O}$ in a population, this only implies that $\mathrm{T}$ causes $\mathrm{O}$ in at least some members of that population. ${ }^{2}$ Reporting average results can be misleading if the population is not causally homogeneous.

Ideally, one should try to identify the specific subgroup of responders. The authors could consider analysing their data further by looking at demographic factors such as age, because younger people are less likely to respond, ${ }^{3}$ and the presence of particular symptoms such as anxiety, which might adversely influence outcome as well, ${ }^{4}$ if these data are available. However, it is unlikely that one can identify the subgroups of responders with $100 \%$ accuracy with these additional data.

More generally, Thase et al's results show that by reporting average results, important findings from RCTs might be missed. Reporting binary outcomes such as number or percentage of patients improved or in remission should be encouraged in psychiatric research, even if the primary outcome variable is considered to be a continuous interval variable. This applies to all RCTs with non-homogeneous populations. Unfortunately, CONSORT requirements do not make this compulsory at the moment. $^{5}$

1 Thase ME, Larsen KG, Kennedy SH. Assessing the 'true' effect of active antidepressant therapy $v$. placebo in major depressive disorder: use of a mixture model. Br J Psychiatry 2011; 199: 501-7.

2 Cartwright N. Are RCTs the gold standard? BioSocieties 2007; 2: 11-20.

3 Tsapakis EM, Soldani F, Tondo L, Baldessarini RJ. Efficacy of antidepressants in juvenile depression: meta-analysis. Br J Psychiatry 2008; 193: 10-7.

4 Coryell W, Fiedorowicz JG, Solomon D, Leon AC, Rice JP, Keller MB. Effects of anxiety on the long-term course of depressive disorders. Br J Psychiatry 2012; 200: 210-5.

5 Schulz KF, Altman DG, Moher D. CONSORT 2010 statement: updated guidelines for reporting parallel group randomized trials. Ann Int Med 2010; 152: $1-8$

Dieneke Hubbeling, Consultant Psychiatrist, Wandsworth Crisis and Home Treatment Team, South West London and St George's Mental Health NHS Trust, 16 Glenburnie Road, London SW17 7DJ, UK. Email: dieneke@doctors.org.uk

doi: 10.1192/bjp.200.5.430

Long-term psychodynamic psychotherapy in complex mental disorders: update of a meta-analysis. BJP, 199, 15-22. Figure 2 (p. 19): total effect size CI should read 0.26 to 0.83 .

Predicting the development of schizophrenia: authors' reply (letter). BJP, 200, 255. Jefter Chuma is Clinical Studies Officer with NIHR Mental Health Research Network (North West Hub), and Prem Mahadun is Consultant Psychiatrist with the Trafford Crisis Resolution and Home Treatment Team. 